ARTICLE

DOI: $10.1038 / s 41467-018-04102-1$

\title{
Manipulation of insulin signaling phenocopies evolution of a host-associated polyphenism
}

\author{
Meghan M. Fawcett ${ }^{1}$, Mary C. Parks ${ }^{1}$, Alice E. Tibbetts ${ }^{1}$, Jane S. Swart ${ }^{1}$, Elizabeth M. Richards (D) ${ }^{1}$, \\ Juan Camilo Vanegas', Meredith Cenzer (10 ${ }^{2}$, Laura Crowley ${ }^{1,3}$, William R. Simmons ${ }^{1,4}$, Wenzhen Stacey Hou ${ }^{1} \&$ \\ David R. Angelini (iD ${ }^{1}$
}

Plasticity, the capacity of an organism to respond to its environment, is thought to evolve through changes in development altering the integration of environmental cues. In polyphenism, a discontinuous plastic response produces two or more phenotypic morphs. Here we describe evolutionary change in wing polyphenism and its underlying developmental regulation in natural populations of the red-shouldered soapberry bug, Jadera haematoloma (Insecta: Hemiptera: Rhopalidae) that have adapted to a novel host plant. We find differences in the fecundity of morphs in both sexes and in adult expression of insulin signaling components in the gonads. Further, the plastic response of ancestral-state bugs can be shifted to resemble the reaction norm of derived bugs by the introduction of exogenous insulin or RNA interference targeting the insulin signaling component encoded by FoxO. These results suggest that insulin signaling may be one pathway involved in the evolution of this polyphenism, allowing adaptation to a novel nutritional environment.

\footnotetext{
${ }^{1}$ Department of Biology, Colby College, 5734 Mayflower Hill, Waterville, ME 04901, USA. ${ }^{2}$ Department of Entomology, University of California, Davis, One Shields Avenue, Davis, CA 95616, USA. ${ }^{3}$ Department of Genetics and Development, Columbia University Medical Center, 1130 Street Nicholas Avenue, Room 208B, New York, NY 10032, USA. ${ }^{4}$ National Human Genome Research Institute, 49 Convent Drive, Bethesda, MD 20892, USA. Correspondence and requests for materials should be addressed to D.R.A. (email: david.r.angelini@gmail.com)
} 
$\mathrm{P}$ henotypic plasticity is a non-genetic source of biological variation $^{1-3}$. Nevertheless, it arises from developmental systems, which are produced by gene products and subject to evolution ${ }^{2-6}$. The average response of individuals in a population to a given environmental influence describes their reaction norm $^{7,8}$. Despite identification of regulatory mechanisms for some reaction norms ${ }^{9-16}$ and documentation of the evolution of plasticity in many systems ${ }^{17-26}$, mechanisms underlying reaction norm evolution in wild populations remain poorly understood. It remains unclear how genetic pathways that enable phenotypic sensitivity to the environment might change with evolution to modify a plastic response.

Wing polyphenism occurs in many insects, including in many true bugs (Hemiptera). In these species, adults develop with shortened wings and non-functional flight muscles. In most species, wing morphs are determined by environmental conditions, such as nutrition, or by a combination of genetic and environmental influences on development $t^{5,11,27}$. Although several developmental genetic pathways are associated with body size and organ growth regulation ${ }^{28}$, less is known about growth regulation in the context of polyphenism ${ }^{29,30}$. Recent studies of wing polyphenism in the brown planthopper Nilaparvata lugens ${ }^{31}$ and other species ${ }^{32-34}$ have identified a role for insulin signaling in the specification of wing morphs in these species.

The soapberry bug Jadera haematoloma (Hemiptera: Rhopalidae) is a promising model for integrative study into the regulation and evolution of a reaction norm. This insect exhibits a nutritionally regulated wing polyphenism, which has diverged among populations only in recent decades. Soapberry bugs are native to coastal dry hammock forest, along the Caribbean Sea from the US Gulf of Mexico to South America (Supplementary Fig. 4). Jadera feed on several plants of the soapberry family, native to the United States, including Cardiospermum sp. in Florida $^{35}$. Since about 1950 , a population of J. haematoloma has adapted to a novel host plant, the introduced goldenrain tree (Koelreuteria sp. $)^{36-38}$. Therefore, traits associated with the introduced host are likely to be recently derived. Cardiospermum are perennial vines with relatively low but consistent seed production. In contrast, Koelreuteria are large trees that produce abundant seeds during a 3- to 4-month period. Compared with Cardiospermum, Koelreuteria seeds have a higher proportion of lipids and lower concentration of protein ${ }^{38}$. Many adaptations in morphology and life history have been documented for hostassociated ecotypes of $J$. haematoloma ${ }^{36-39}$. The Koelreuteriaadapted ecotype is now widely distributed and expanding in the temperate United States, whereas populations resembling the ancestral state remain associated with native Cardiospermum vines in the Florida Keys (but see ref. ${ }^{40}$ ).

Adult $J$. haematoloma of both sexes exhibit distinct wing morphs. Long-wing morphs have complete wings and functional flight muscles, whereas short-winged individuals are brachypterous, lack flight muscles, and are incapable of flight. The wings of each morph differ in their overall size, shape, and venation, and this variation is determined by environmental influences rather than genetic factors. Insect polyphenisms are typically mediated by one or more factors, including juvenile nutrition or density ${ }^{5,27}$. Previous work has suggested that J. haematoloma morphs are determined by juvenile nutrition, with a positive correlation between food level and frequency of the short-winged morph $^{41,42}$. Host plants with different phenology present soapberry bugs with distinct nutritional environments ${ }^{38}$, potentially selecting for differences in wing morph frequencies or reaction norms.

Here we present evidence that J. haematoloma wing polyphenism is adaptive in females, but maladaptive in males of the native host ecotype. This sexual conflict is partially resolved in the derived Koelreuteria ecotype, in which the reaction norm offset has evolved to make short-winged individuals more common. The frequency of morphs is determined nutritionally and associated with differences in the expression of genes encoding insulin signaling components. Manipulation of the insulin signaling pathway alters this reaction norm, phenocopying its evolution in natural populations.

\section{Results}

Wing morphology. The range of wing phenotypes in J. haematoloma was characterized using linear and geometric morphometrics (Fig. 1 and Supplementary Figs. 1-3) ${ }^{43}$. Each sex has a bimodal distribution of wing lengths (Fig. 1h). Wing morphs are significantly different in wing length (permutation analysis of variance (ANOVA) with morph and sex as factors, $p<2.2 \times$ $\left.10^{-16}\right)$. Wing morphs also differ dramatically in their static allometry $^{1}$. Compared with head width (a proxy for body size), wing lengths of the two morphs have significantly different scaling relationships (Supplementary Fig. 1; analysis of covariance test for homogeneity of slopes $F_{1,162}=835.3, p=7.96 \times 10^{-66}$ ). Host-associated ecotype was not a significant factor in this relationship. Despite distinct differences in adult wing length among morphs, the size of juveniles or their external wing pads does not indicate an individual's future morph (Supplementary Fig. 3).

Morphs differ significantly in wing shape as well as wing size. Variation in wing shape was characterized using landmark-based geometric morphometric analysis (Fig. 1a, b) ${ }^{43-45}$. Morphs differ significantly in their shape (Fig. 1i; permutation-based Procrustes ANOVA $^{44}$ with morph and sex as factors, $F_{1,183}=335.1$, $\left.p<10^{-4}\right)$. The wings of each morph differ most obviously in the shape of the distal wing region known as the membrane. Therefore, we reasoned that morph-specific cues might vary in their influence over different regions of the wing, highlighting anatomical and developmental modules. Several modularity hypotheses were tested by dividing the wing along proximal-distal and anterior-posterior axes, and evaluating the covariance ratio coefficient produced by comparisons within and between the proposed modules. As expected, the membrane and more proximal regions of the wing displayed significant modularity (covariance ratio test ${ }^{45}, p<0.05$; Supplementary Note 1), whereas other groupings of wing landmarks did not (Fig. 1c).

Subjectively, short-wing morphs display a wide range of wing appearances, whereas long-wing morphs have more consistent appearance. Procrustes variance ${ }^{44}$ was significantly greater in short-wing morphs (difference in Procrustes variance $=0.00867$; randomized residual permutation, $p<10^{-6}$ ). We also compared the wing shape disparity of each morph between ecotypes. Shortwing morphs differed slightly in their disparity between ecotypes (difference $\left.=3.784 \times 10^{-3}, p=0.0419\right)$ with greater disparity in Koelreuteria short-wing shapes. Among long-wing morphs, the difference in wing shape disparity for ecotypes was much greater $\left(\right.$ difference $\left.=1.093 \times 10^{-3}, p=1.69 \times 10^{-3}\right)$, with lower variation among Cardiospermum-associated long-wing bugs.

Wing morph frequencies vary in the wild. To explore potential differences in wing polyphenism, we surveyed the frequency of each morph at numerous sites across the US range of J. haematoloma, including populations living on native and introduced hosts (Supplementary Fig. 4). The frequency of wing morphs varied widely, with some sites having only long-wing (11 of 64) or short-wing bugs ( 3 of 64 ), whereas most sites had both morphs. The geographic location of sites was not a significant factor in explaining variation in morph frequencies. However, morph ratios differed significantly among the most heavily sampled host plants: Cardiospermum corindum, a native host, and two 

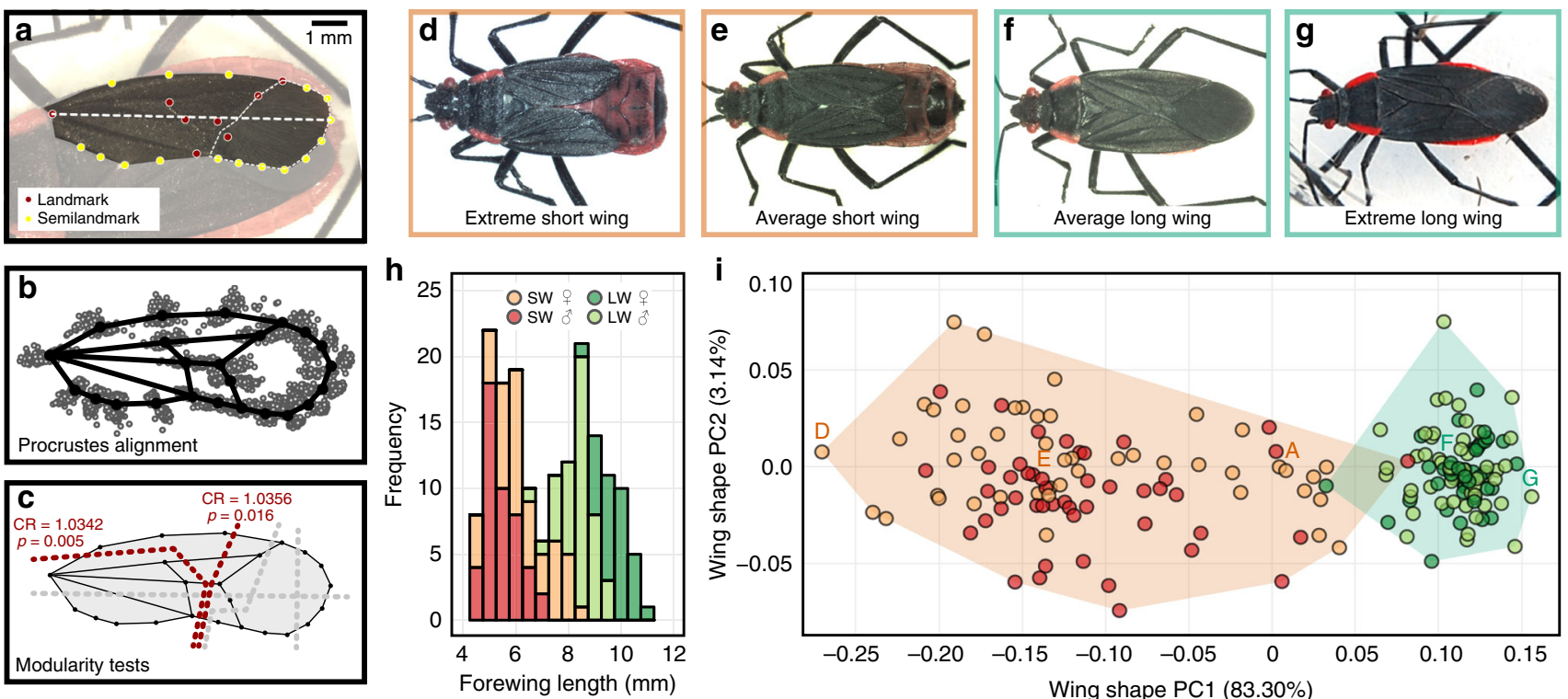

Fig. 1 Two morphs of J. haematoloma differ most noticeably in the length and shape of the forewings. a Landmarks were defined at 24 locations across the wings, including 8 anatomical landmarks and 16 sliding semilandmarks. The dotted white line indicates the measurement of wing length. $\mathbf{b}$ Generalized Procrustes alignment of wings from 189 specimens. c Wings exhibit proximal and distal modules. Significant modular boundaries are marked by red dotted lines. Gray dotted lines demarcate alternative hypotheses that did not display significant modularity. $\mathbf{d}$ - $\mathbf{g}$ Extreme and average representatives of each wing morph, as determined from the first PC axis in $\mathbf{i}$. h The distribution of wing length frequencies in $168 \mathrm{~J}$. haematoloma adults is distinctly bimodal. Females are larger on average than males, but both sexes display polyphenic variation. Colors indicate sex and morph of sampled individuals. i Plot of 189 Procrustesaligned specimens from $\mathbf{b}$ in tangent space, oriented to the first two principal component axes. The percentage of wing-shape variation along each axis appears in parentheses. Convex hulls highlight the disparity of each morph. Wing shape varies significantly by morph. Males and females do not differ significantly in wing shape. The individuals shown in $\mathbf{a}$ and $\mathbf{d}-\mathbf{g}$ are labeled above their corresponding positions in morphospace

introduced hosts, Koelreuteria elegans and Koelreuteria paniculata (permutation ANOVA, $p<2.2 \times 10^{-16}$ ). Short-wing morphs were significantly more common on $K$. paniculata compared with each of the other two host plants (permutation test with Bonferroni correction; vs. C. corindum, $p=1.33 \times 10^{-3}$; vs. K. elegans, $\left.p=8.12 \times 10^{-5}\right)$.

Mendelian inheritance does not explain morph frequencies. To test whether J. haematoloma wing morphs are determined genetically, we made 32 controlled crosses with all morph combinations (Supplementary Table 2). For each cross, we tested whether the resulting adult $\mathrm{F}_{1}$ morph ratio matched any Mendelian ratio $(0: 1,1: 3,1: 1,3: 1$, or 1:0 short wing to long wing) or the prediction of a recessive lethal short-wing allele (2:1) using Fisher's exact test with Bonferroni correction. These results were then compared for compatibility with Mendelian dominance of the long-wing or short-wing phenotypes and with the possibility of a recessive lethal allele. No Mendelian model consistently fit the data (Supplementary Table 2), consistent with a past study ${ }^{41}$ and supporting the conclusion that wing morphs in J. haematoloma are not primarily based on genetic factors.

Nutrition-dependent wing polyphenism varies among ecotypes. As wing morph frequencies vary among wild populations on host plants with different phenology and resource availability (Supplementary Fig. 4), we wished to test whether ecotypes vary in their norms of reaction to food availability. Previous work has suggested that the Cardiospermum ecotype was more responsive to environmental conditions than soapberry bugs living on introduced Koelreuteri ${ }^{41}$. In field surveys, morph ratios are likely to be affected by environmental cues, genetic background, as well as selective forces that might bias the adults counted in field surveys. Therefore, we raised J. haematoloma cohorts in the lab under a wide range of food and conspecific density conditions, and surviving adults were scored by morph (Fig. 2a).

Using logistic regression, we compared models with predictive factors of seed number, the initial hatchling number in the cohort, and ecotype, alone, in combination, and allowing for interactions (Supplementary Table 3). We also considered null models predicting all short-wing or all long-wing morphs. This dataset included 3490 sampled adults from 133 independent treatments. An individual's morph was best predicted by a model incorporating seed number, cohort size, and their interaction, as well as the bugs' host ecotype. Each of these factors was a significant influence on the model fit (Supplementary Table 4 and Supplementary Fig. 5). Increasing seed number and lower conspecific competition (smaller cohorts) were correlated with increased frequencies of the short-wing morph (Fig. 2;0.70\% increased odds per seed; $0.95 \%$ decreased odds per bug). Compared with Cardiospermum ecotypes, populations of the Koelreuteria ecotype were 10-14 times more likely to be short winged (95\% confidence on odds ratio). Models were tested in logistic regression for each ecotype alone, where seed number and cohort size were also found to be significant predictors of morph frequency (Supplementary Tables 5-8). These analyses reveal that ecotypes differ primarily in the offset of their reaction norms ${ }^{46}$, with Koelreuteria ecotypes having a lower nutritional threshold for short-wing development.

In the previous analysis, bugs were raised on seeds of the host plant associated with their ecotype. Therefore, we tested for potential effects that the host plants might have on morph determination by cross-rearing juveniles on non-natal seeds (Fig. 3). The resulting offspring were examined using permutation-based factorial ANOVA, considering the effects of ecotype and seed species on the frequency of short-wing morphs. Although the influence of ecotype on morph frequencies was significant (overall model main effect for ecotype, $F_{1,94}=47.24$, 
a
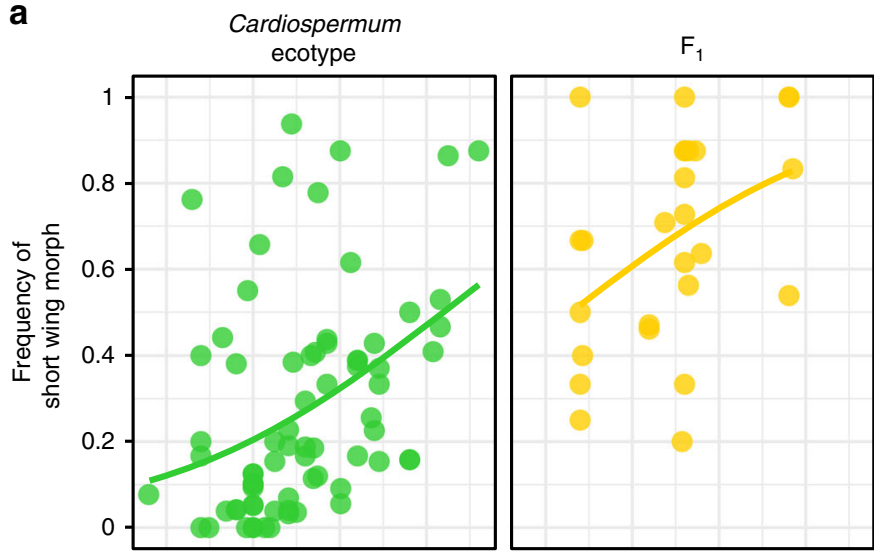

b

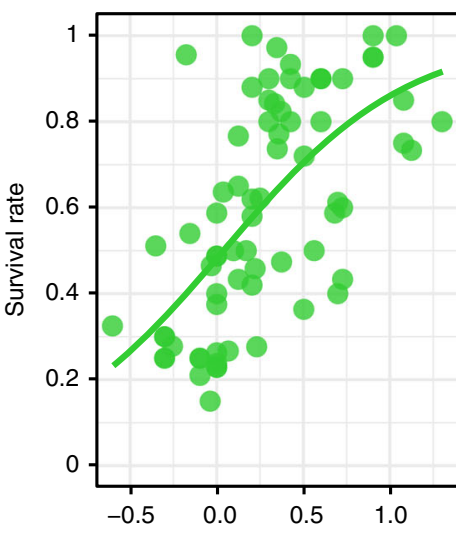

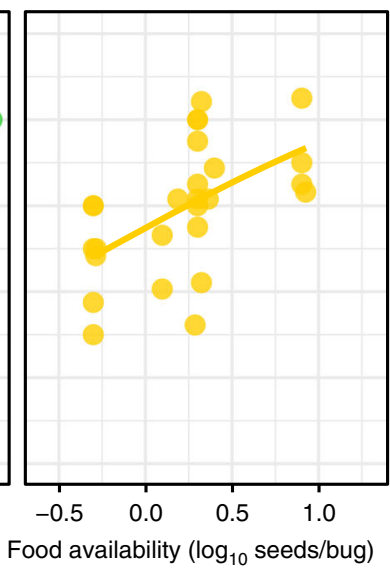
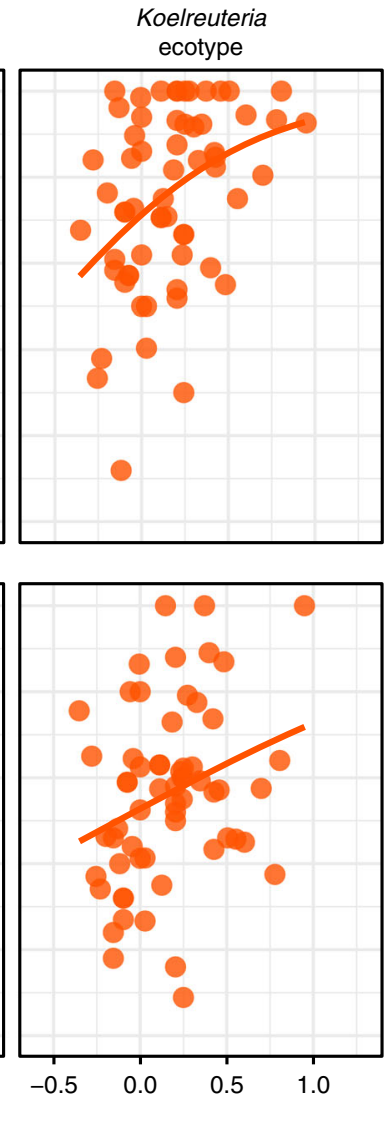

Fig. 2 The frequency of wing morphs $\mathbf{a}$ and juvenile survival $\mathbf{b}$ correlates with juvenile food availability for two host-adapted ecotypes of $J$. haematoloma. The reaction norms vary among ecotypes. The ancestral Cardiospermum ecotype has a lower offset than the more recently adapted Koelreuteria ecotype. Each point represents a cohort raised under varying conditions of food availability. Survival rate also increases with food availability, but does not vary among ecotypes. The reaction norm of $F_{1}$ ecotype hybrids strongly resembled the Koelreuteria ecotype. This dataset includes 3490 sampled adults from 133 treatments. Lines indicate logistic regression

$p<2 \times 10^{-16}$ ), cross-reared bugs of each ecotype did not differ significantly in morph frequency (main effect of seed species, $F_{1,94}=0.635, p=0.38$; Fig. 3a, compare left and right panels for each ecotype). Interestingly, survival to adulthood was reduced for bugs raised on non-natal seeds (Fig. 3b). This decrease in survival was significant for Koelreuteria-adapted bugs raised on Cardiospermum seeds (overall model interaction of ecotype and seed species, $F_{1,94}=6.85, p=6.00 \times 10^{-3}$; two-sample permutation test for Koelreuteria ecotype, $Z=-2.90, p=3.72 \times 10^{-3}$ ).

The response of $\mathrm{F}_{1}$ hybrids from crosses of Cardiospermum and Koelreuteria ecotypes suggest that host plants can exert influence on wing morph frequency. Among $F_{1}$ hybrids, the host seed species used for rearing was a significant factor in wing morph ratios (exact permutation, $p=9.12 \times 10^{-4}$ ). However, the direction of this effect is opposite that seen for host-associated ecotypes, with more long-wing adults produced when hybrid bugs are raised on K. paniculata seeds. Survival of hybrids was higher on both seed species than for cross-reared parental ecotypes, although this difference is marginal (exact permutation, $p=$ 0.0714 ). The reduced survival of hatchlings on non-natal seeds may present an obstacle to the colonization of new host plant species in the wild. However, the high survival of ecotype hybrids on both plants suggests that contemporary gene flow may still be possible among ecotypes ${ }^{40-42}$.

Sexual conflict in wing polyphenism varies by ecotype. As J. haematoloma ecotypes vary in their polyphenic reaction norms
(Fig. 2), we sought to test whether the reproductive advantage predicted for short-winged females might vary among ecotypes. On average, for each ecotype, wild-caught short-winged females laid eggs faster than long-winged females, and this difference was significant among Koelreuteria ecotypes (Fig. 4a; Wilcoxon test with Bonferroni adjustment, $W=27, p=0.00429)$. Among the Cardiospermum ecotype, the egg-laying rate was higher on average in short-winged females (Fig. 4a). Increased fecundity for short-winged females is consistent with the classic models of a dispersal-fecundity polyphenic trade-off ${ }^{5,47}$.

We also examined lifetime egg production from females in laboratory crosses (Fig. 4b, c). Effects on fecundity were tested using permutation-based factorial ANOVA, considering the morph and ecotype of each parent and potential interactions among morphs. Female morph was not a significant factor, although crosses of Koelreuteria ecotypes involving short-winged females did produce marginally more eggs (Fig. 4c; $Z=-1.55$, $p=0.12$ ). Females raised in the lab have access to energy stores unavailable to wild females and these lab-reared long-winged females do not expend energy flying before being able to mate. For these reasons, polyphenism may still be adaptive in Cardiospermum ecotype females. Seed resources are typically much more widely distributed in Cardiospermum habitats ${ }^{36}$, suggesting a greater advantage to the dispersal ability conferred by flight.

It has been unclear whether wing polyphenisms in different species might be adaptive for males, or if male flightlessness 

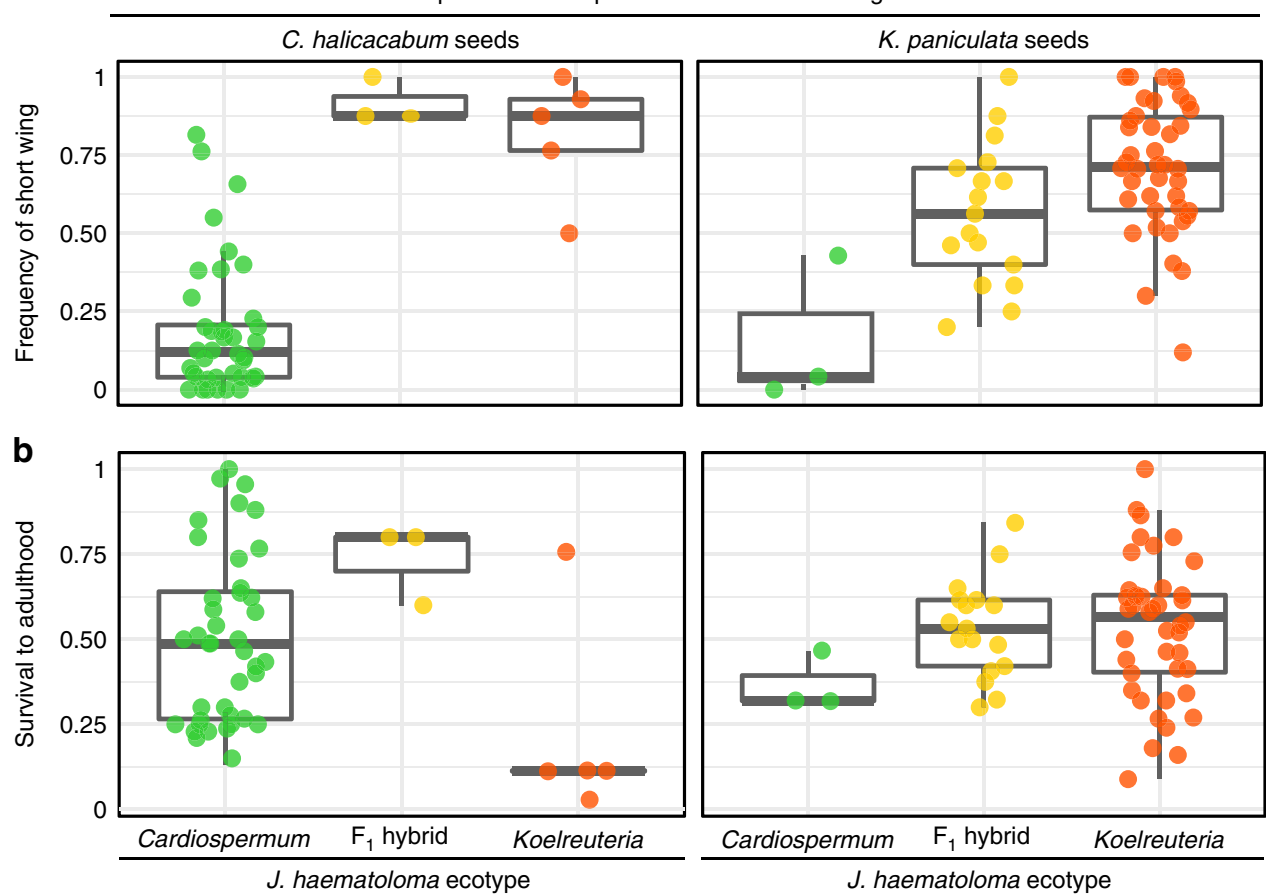

Fig. 3 The effects of cross-rearing J. haematoloma on non-natal host plant seeds. a Wing morph frequencies were not significantly affected by cross-rearing. Treatments included in the natal groups (e.g., Cardiospermum bugs raised on Cardiospermum seeds) are taken from the full dataset with values of food availability comparable to the cross-reared treatments. b Survival to adulthood was reduced when bugs were raised on seeds of non-natal hosts. The distribution of values is shown by Tukey's plots: boxes demarcate the upper and lower quartiles, whereas the heavy bar indicates the median value. Whiskers extend to 1.5 times the interquartile range or the most extreme value. Each superimposed point represents one cohort of multiple bugs

a

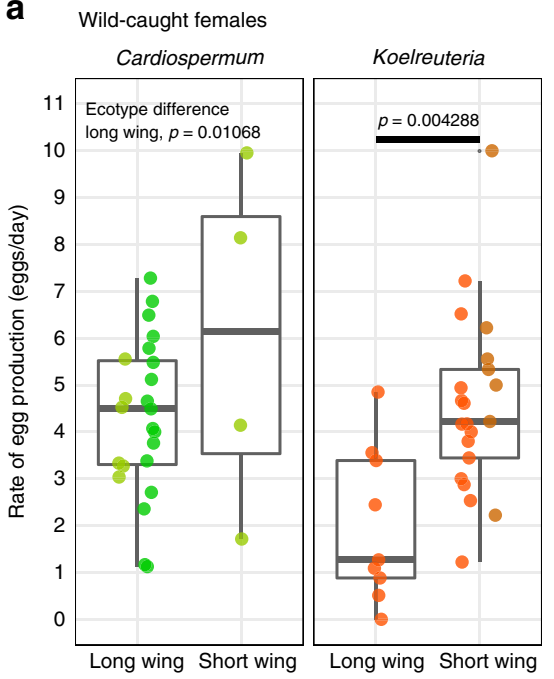

b

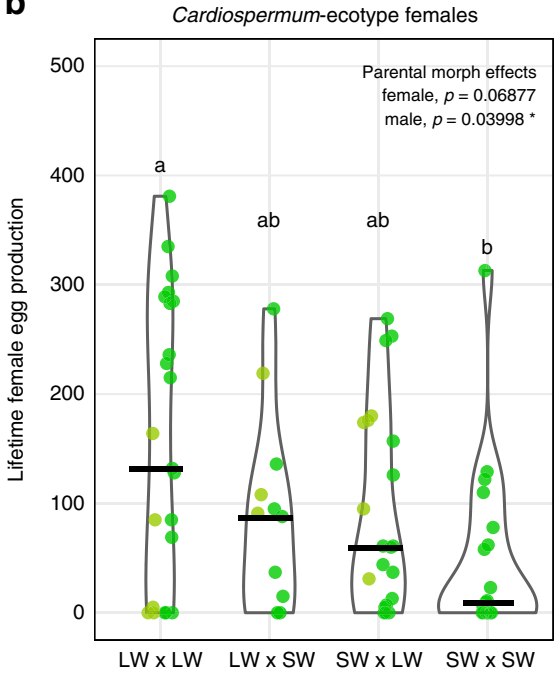

C

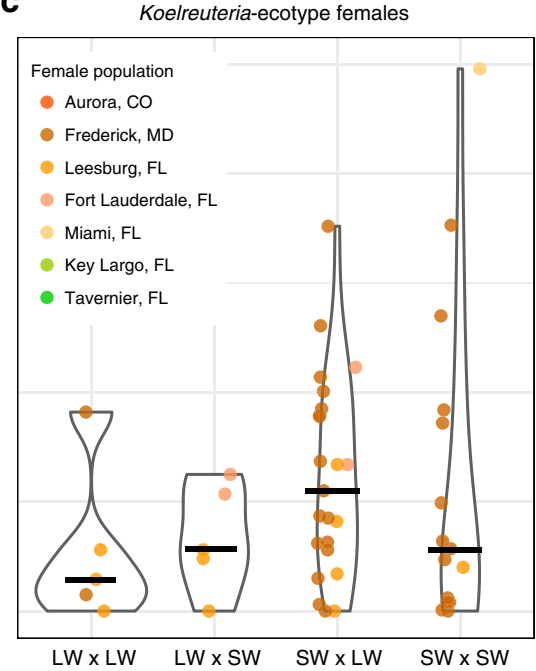

Fig. 4 Reproductive output differs by morph and ecotype. a The rate of egg production among wild-caught females of different morph and ecotype is represented by individual points, colored by population of origin. Tukey's box-whisker plots summarize the dispersion of values and the median for each group as in Fig. 3. b, c Lifetime female egg production from laboratory crosses of different morph and ecotype. The results of individual crosses are denoted by points, colored by population of origin. Violin plots summarize the dispersion of cross results. Heavy bars indicate the median outcome. Significant global effects, as determined by permutation-based ANOVA, are superimposed on the plot. Letters indicate significant difference among cross types based on post-hoc pairwise permutation tests. Abbreviations: LW, long-winged; SW, short-winged

represents a sexual conflict produced by pleiotropy ${ }^{29,47}$. Surprisingly, for the Cardiospermum ecotype, crosses produced significantly fewer offspring when males were short winged (Fig. $4 \mathrm{~b}$; permutation with Bonferroni correction, $Z=2.326, p=0.0400$ ). However, this effect was not found for the Koelreuteria ecotype, where short-wing bugs of both sexes are much more common in the wild. Differences in male fertility were not due to differences in the size of testes or accessory glands (Supplementary Fig. 6).

These results suggest a sexual conflict in wing polyphenism. Polyphenism appears to be adaptive for females, allowing faster egg production in high-nutrient environments. However, for populations on Cardiospermum, the polyphenism appears to be 
maladaptive for males, which suffer reduced fertility when short winged. Short-winged bugs are much more common in populations living on $K$. paniculata, which appear to have evolved a resolution to this sexual conflict, allowing more equal fitness for males of both morphs.

Dominance of the derived reaction norm. In order to explore the underlying genetic basis for ecotype differences in nutritional reaction norms, crosses were made with virgin adults from Tavernier, Florida, which feed on C. corindum and have a reaction norm offset favoring long-wing morphs, and Frederick, Maryland, which live on K. paniculata and are more frequently short winged at comparable levels of juvenile food availability. Reciprocal crosses showed no evidence of maternal effects. Hybrid cohorts were raised with varying numbers of seeds.

If reaction norms have evolved by changes in mostly additive genetic variance, then hybrids should display an intermediate reaction norm compared with the parental ecotypes. Strong dominance, in which the $\mathrm{F}_{1}$ reaction norm resembles one of the parents, would be evidence that population differences in plasticity are produced by genetic differences with strong nonadditive effects. The morph frequencies of $F_{1}$ cohorts and their norm of reaction strongly resembled the Koelreuteria ecotype (Fig. 2, middle). We used a logistic model of morph determination in hybrid and parental populations to examine the effect of genetic background on the reaction norm (Supplementary Table 9). Compared with $F_{1}$ hybrids, the odds that a bug will be short winged are four to seven times lower for the Cardiospermum parental population ( $95 \%$ confidence on odds ratio). In contrast, bugs from the Koelreuteria parental population were not significantly different in their predicted morphs from the $\mathrm{F}_{1}$.

Together, these results suggest that although the frequency of wing morphs is environmentally determined, the threshold for that response is determined by the genetic background. Responses of the ecotype hybrids demonstrate that this genetic difference may have evolved through changes in relatively few genes with large effect and strong dominance.

Insulin pathway components in the soapberry bug. Insulin signaling mediates nutrition-dependent growth in various contexts ${ }^{34,48,49}$, making it a candidate mechanism for the evolution of nutrition-dependent polyphenism ${ }^{32}$. Functional tests in the planthopper $N$. lugens have also implicated the activities of paralogous insulin receptors in wing morph development in that species $^{31}$. We assembled transcriptome sequences for each $J$. haematoloma ecotype. This allowed the identification of one-toone orthologs for most insulin pathway components. Two genes encoding orthologs of the Drosophila insulin receptor were identified in J. haematoloma (Supplementary Fig. 7), as well as from the boxelder bug Boisea trivittata (Rhopalidae) and the milkweed bug Oncopeltus fasciatus (Lygaeidae). One insulin receptor ortholog (InR1) was also isolated from a species of monomorphic soapberry bug, J. sanguinolenta.

In order to explore whether insulin signaling components differ in their expression by morph, we measured transcript abundance in nascent adults using quantitative PCR (qPCR). At this stage, bugs are undergoing sexual maturation, making gene expression relevant to fecundity. The ovaries of nascent adult females showed differences in the expression of the insulin receptor genes by ecotype (Supplementary Fig. 8b, c). The relative expression of FoxO, a transcription factor downstream of the insulin signaling pathway ${ }^{48}$, also differed by morph among females of the Cardiospermum ecotype (Supplementary Fig. 8a). In the absence of insulin-like peptides, FoxO inhibits expression of genes related to protein synthesis and cell division ${ }^{50,51}$, while promoting its own transcription and that of the insulin receptor ${ }^{52}$.

Manipulation of insulin signaling alters the reaction norm. We tested the influence of insulin signaling on J. haematoloma development and wing morph specification by treating fourth instars with exogenous insulin ${ }^{53,54}$ or via RNA interference (RNAi) targeting the insulin receptor genes (InR1 and InR2), two signal transducers, chico and $A k t$, and FoxO. These experiments were conducted with juveniles raised under a range of food regimes, to examine developmental genetic control of wing morph reaction norms (Fig. 5) and wing shape (Fig. 6). RNAi knockdown was verified using quantitative reverse transcriptasePCR (qRT-PCR) (Supplementary Fig. 9).

Activation of insulin signaling, by introduction of exogenous insulin or knockdown of FoxO, phenocopied the evolution of reaction norms from the high-threshold Cardiospermum host ecotype to resemble the Koelreuteria ecotype, which has a much lower threshold for short-wing development (Fig. 5a). FoxO RNAi significantly altered the reaction norm for wing morph frequencies such that they were 10-38 times more likely to be short winged than unmanipulated bugs (95\% confidence intervals on odd ratio from logistic regression; Supplementary Table 12). This increase in the likelihood of the short-wing morph was significant in comparison with nonspecific green fluorescent protein (GFP) double-stranded RNA (dsRNA) controls (Wald's test, $z=5.69, p=1.31 \times 10^{-8}$; Supplementary Table 13). Injection of roughly $0.5 \mathrm{ng}$ insulin per $\mathrm{mg}$ body weight had a similar effect, increasing the odds of short-wing development by four to nine times, a significant difference from control injections $(z=$ $3.51, p=4.46 \times 10^{-4}$; Supplementary Table 13). Similar effect sizes for insulin and FoxO RNAi were found within the Cardiospermum ecotype (Supplementary Table 14), although these treatments did not significantly influence wing morph frequencies in Koelreuteria ecotypes, where short-wing bugs are already common. Conversely, reduction of insulin signaling by RNAi targeting InR1 and InR2 simultaneously resulted in marginally lower frequencies of short-wing bugs among Koelreuteria ecotypes $(z=-1.818, p=0.0691)$. In Drosophila, InR activation inhibits FoxO which normally acts to suppress target genes related to growth ${ }^{49,51}$. Therefore, these results are consistent with a model in which increased food consumption activates insulin signaling, potentially in concert with other pathways, to specify development of the short-wing morph.

Although FoxO RNAi during late juvenile development increases the frequency of short-wing morphs, FoxO expression is higher in ovaries and dorsal thorax of nascent adult short-wing morphs, compared with long-wing bugs (Supplementary Fig. 8). This apparent paradox likely reveals dynamic expression and function of FoxO between these different life stages.

Targeting other components of the insulin pathway by RNAi failed to alter development in obvious ways. Knockdown of chico and Akt (Supplementary Fig. 11), which encode intracellular transducers of insulin signaling, extended the fifth instar by five to ten times its normal duration, greatly extending their normal lifespans. However, this result precluded a determination of any influence these genes may have on morph specification.

FoxO RNAi biases the development of wing shape. As FoxO RNAi significantly increased the frequency of short-wing morphs in Cardiospermum ecotypes (Fig. 5 and Supplementary Tables 13, 14), we examined whether knockdown of this gene might also influence the shape of wings. We utilized the same geometric morphometric analysis applied to unmanipulated bugs (Fig. 1) on 


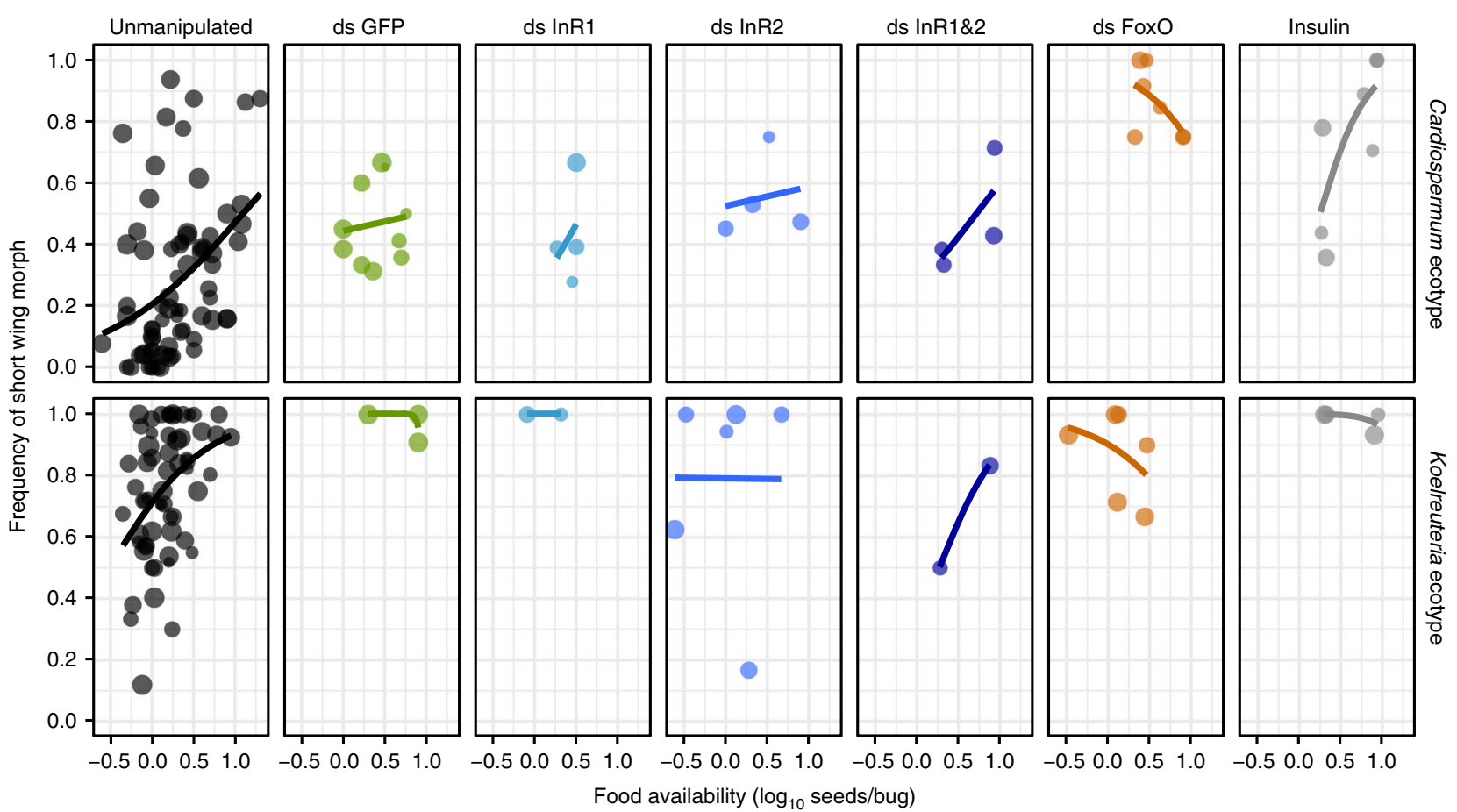

Fig. 5 Manipulation of insulin signaling alters wing morph reaction norms. Effects of RNAi treatment on the frequency of short-wing morphs in Cardiospermum (top row) and Koelreuteria (bottom row) ecotypes. Unmanipulated bugs (left column) differ in their reaction norms based on ecotype. In the Cardiospermum ecotype, RNAi targeting FoxO or injection of exogenous insulin significantly alter the reaction norm, making it resemble the Koelreuteria ecotype. Knockdown of both insulin receptor genes in the Koelreuteria ecotype produces a marginal decrease in the rate of short-winged adults. Each point represents a treatment cohort of multiple individuals. Point size indicates relative survival rate. These data include 4218 scored adults ( 820 from RNAi) in 187 cohorts (59 RNAi treatments)
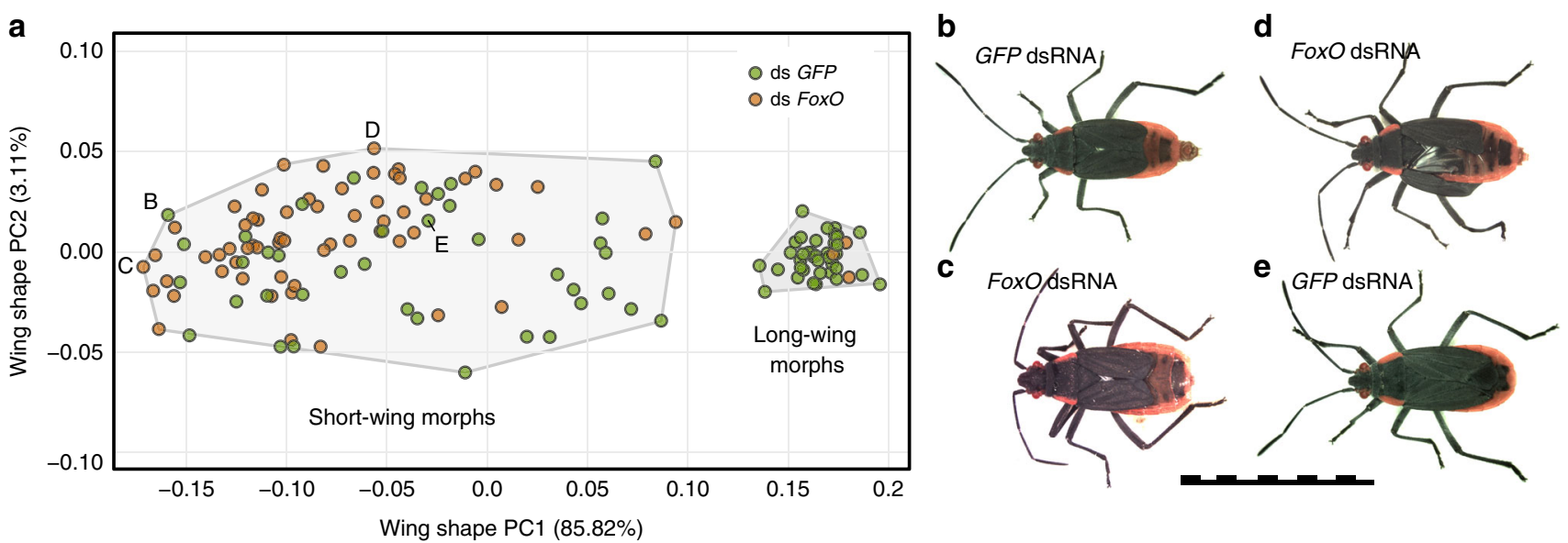

Fig. 6 Knockdown of FoxO biases short-wing shapes. a Principle component analysis of wing shapes from GFP and FoxO RNAi specimens. RNAi treatment has a significant effect on wing shapes, with FoxO RNAi skewing them towards the upper left. $n=141$. b-e Specimens representing different areas of the morphospace in panel A. FoxO RNAi specimens resemble extreme individuals from the control treatment. Control specimens with extreme $\mathbf{b}$ and average e wing shapes are shown in photographs, as are FoxO RNAi specimens with extreme positions on PC1 $\mathbf{c}$ and PC2 $\mathbf{d}$. For each specimen the shape of the right (top) wing was analyzed. Warp grids representing the wing shapes of these and other specimens appear in Supplementary Fig. 14 . The scale bar is $1 \mathrm{~cm}$

77 GFP dsRNA-treated control specimens and 64 FoxO RNAi individuals. Principal component analysis of RNAi wing shapes (Fig. 6a) placed short- and long-winged bugs in a similar relationship in morphospace, compared with unmanipulated bugs (Fig. 1i). However, RNAi treatment had a significant effect on wing shape overall (Procrustes ANOVA, $F_{1,138}=8.66, p<10^{-4}$ ) and among short-wing morphs $\left(p<2 \times 10^{-4}\right.$ with Bonferroni adjustment). FoxO RNAi short-wing shapes occupied a similar region of morphospace as control wings, but the FoxO-knockdown group was biased toward extreme short-wing shapes. These wings were more severely reduced in the distal membrane region (low PC1 values in Fig. 6a, Fig. 6c, d compared with 6e, Supplementary Fig. 14) and narrower in the anterior-posterior dimension (high PC2 values). RNAi treatment did not have a 
significant effect on long-wing shapes; however, the power of this inference is limited by the small number of long-wing FoxO specimens $(n=3)$ in the shape analysis.

Other appendages were also examined for allometric effects of RNAi targeting FoxO and InR1. No significant effects were found for the relative lengths of antennae, the labrum, labium (beak), femora of the legs, and wings (Supplementary Note 1). However, body length and pronotum width were affected by knockdown of these genes, with InR1 RNAi decreasing relative size and FoxO RNAi increasing relative size (Supplementary Fig. 12).

\section{Discussion}

Genes act in an environmental context to determine phenotypes and the mechanisms by which environmental conditions exert an influence on development are subject to evolution $3,19,22,23,25,46$. Therefore, reaction norms can evolve over time, vary among populations, and adapt under the influences of selection. Here we have examined nutritionally dependent plasticity in the determination of wing morphs in the soapberry bug J. haematoloma, characterizing morphology, population-level variation in plasticity, and fitness implications, as well as developmental mechanisms.

Variation in the length and shape of J. haematoloma wings is discontinuous (Fig. 1), constituting two discrete morphs. Shortwing morphs display a significantly wider range of wing shapes than long-winged morphs (Fig. 1i). This difference may be nonadaptive, resulting from different degrees of canalization in each developmental trajectory. Alternatively, greater selection on long wings imposed by the need for flight performance may limit their range of shapes. A third explanation for greater short wing disparity is that the differing prevalence of the two morphs exposes them to different selection intensities. This last hypothesis predicts that ecotypes differing in the frequency of a morph should also have reduced disparity in the more common morph. Although short-wing morphs of the two ecotypes differ slightly, but significantly in disparity, long-wing morphs have a highly significant difference in the disparity of their wing shapes. Wingshape disparity is lowest among Cardiospermum-associated longwing bugs, which are the most common morph in that ecotype and which are used by the bugs for flight. These results suggest frequency-dependent selection on wing shapes among long-wing morphs.

There is strong support for an explanation of morph frequencies based on juvenile food availability. Raising juvenile cohorts of J. haematoloma under different resource conditions reveals differing reaction norms for two host-associated ecotypes (Fig. 2). Differences in these reaction norms appear to be genetically based, as evidenced by the persistence of the response when bugs are cross-reared on a non-natal host (Fig. 3) and by dominance of the reaction norm in $\mathrm{F}_{1}$ ecotype hybrids (Fig. 2).

Among ecotype hybrids, a higher frequency of short-wing morphs was produced when bugs were fed Cardiospermum seeds compared with rearing on Koelreuteria seeds. Interestingly, the direction of these hosts' effect on hybrid wing morph frequencies is counter to the normal bias in morph frequencies displayed by the parental ecotypes adapted to those hosts. This suggests that compared with the $\mathrm{F}_{1}$ genetic background, the reaction norms for wing morph determination in each parental, host-adapted ecotype have been modified by selection over the generations since their divergence.

A maladaptive response to nutritional conditions in hybrids may present obstacles to gene flow between ecotypes. However, a recent study of J. haematoloma comparing beak length, egg weight, and developmental time among ecotypes in 2014 with studies ca. 1990 found indirect evidence of maladaptive gene flow from the more numerous Koelreuteria ecotype into Cardiospermum-associated populations in south Florida ${ }^{40,42}$. Poor survival of Koelreuteria ecotypes on Cardiospermum seeds suggests that secondary colonization of the ancestral native host by derived ecotypes may be unlikely without hybridization first.

The Koelreuteria ecotype of J. haematoloma has evolved to be predominantly short winged, which may reflect selection to allocate resources to increasing fecundity in this environment. Koelreuteria are larger plants with a higher abundance of seeds and higher lipid content than Cardiospermum. These factors make dispersal by flight potentially less advantageous on this host ${ }^{47}$, which may explain the differences in ecotype reaction norms. Short-winged females of the Koelreuteria ecotype produce eggs faster than long-winged females (Fig. 4a), helping to maximize fitness in a relatively stable, high-nutrient environment.

It has been unclear whether male wing polyphenism has any adaptive value in J. haematoloma or other species. In contrast, we find that in the Cardiospermum ecotype, where the short-wing morph is rare, short-winged males suffer reduced fitness, as crosses with these sires produced fewer offspring on average (Fig. 4b). Short-winged males are also unable to disperse by flight. These results suggest a sexual conflict, where males maximize fitness consistently with development to the long-winged morph, which provides higher fertility and the opportunity to disperse. Meanwhile females may produce the most surviving offspring by determining their morph based on local food availability. The threshold for short-wing development is lower and short-wing morphs are more common in both sexes of the Koelreuteria ecotype. In this ecotype, the male morph does not influence the fecundity of a cross. Therefore, it is likely that in adapting to Koelreuteria, soapberry bugs evolved a resolution to this sexual conflict, allowing wing polyphenism to remain unlimited by sex without reduced fitness in short-winged males.

Changes in expression (Supplementary Fig. 8) of insulin signaling components in J. haematoloma have accompanied the evolution of the reaction norm for wing morph determination among host ecotypes (Fig. 2). Activation of insulin signaling by exogenous insulin injection or FoxO RNAi can phenocopy this evolutionary change (Fig. 5). Insulin-like peptides are produced in the insect brain 55,56 in response to feeding 55 . In fruit flies, insulin signaling regulates cell proliferation and protein synthesis ${ }^{56,57}$. Therefore, insulin signaling may provide a physiological mechanism by which individual soapberry bugs could gauge their nutritional resources in order to coordinate growth. In other insects, tissue-specific allometries result from the local levels of expression and activation of insulin pathway components ${ }^{33,34,58-60}$. Differences in insulin signaling have been identified in sex-specific allometric differences and in sex-limited plasticity in organ growth ${ }^{33,61}$.

Insulin signaling appears to regulate wing polyphenism in $J$. haematoloma differently than in the brown planthopper $N$. lugens (Hemiptera: Delphacidae) ${ }^{31}$. The cues determining wing morphs in $N$. lugens are not known, although nutrition, stress, and crowding have been suggested as causes ${ }^{62}$. In the planthopper, depletion of InR1 and InR2 cause development of almost entirely short- or long-wing morphs, respectively. Knockdown of $N$. lugens FoxO produces predominantly long-winged planthoppers, the opposite of the effect described here for FoxO RNAi in J. haematoloma. The reason for these dramatic differences is unclear and, although wing polyphenism is widespread among true bugs, rhopalids and delphacids diverged $\sim 320$ mya $^{63}$. Further investigation of polyphenic regulation in these and other species will be necessary to identify themes common to Hemiptera.

The soapberry bug J. haematoloma is a promising model species for the study of polyphenic development and its evolution. 
Our data suggest that in this species, alternative morphs offer trade-offs maximizing either dispersal ability through flight or increased fecundity. Determination of morphs appears to be based on juvenile food availability and this reaction norm has diverged with adaptation to a novel host plant. Wing polyphenism in J. haematoloma is not sex limited. In the ancestral ecotype, short-winged males are rare and appear to suffer reduced fertility. The derived ecotype appears to have evolved a lower offset to the reaction norm, increasing the frequency of short-wing adults of both sexes. Males of this group have similar fitness, regardless of morph, in an apparent resolution to the sexual conflict. Finally, our results demonstrate that artificial activation of insulin signaling can phenocopy evolution of the derived reaction norm. Therefore, we suggest that changes in the insulin signaling pathway may underlie reaction norm evolution in this wild population. The involvement of insulin signaling in diverse developmental contexts, from wing polyphenism in planthoppers $^{31}$ to polyphenism in sexually competitive weapons $s^{33,64}$ supports the hypothesis ${ }^{32}$ that this pathway is a hotspot for the evolution of discontinuous organ growth regulated by nutritional cues.

\section{Methods}

Insect culture. Jadera haematoloma were collected from host plants at several locations, including Tavernier, FL (January 2013 and February 2015), Key Largo, FL (February 2015), Aurora, CO (August 2015), and Frederick, MD (October 2014). Founding cohorts consisted of 50-100 individuals, and multiple cultures from each population-of-origin are maintained with similar numbers each generation. In the lab, soapberry bugs were raised in an incubator at $26^{\circ} \mathrm{C}$ in $3.38 \mathrm{~L}$ plastic terrariums (Carolina Biological Supply Company, Burlington, North Carolina, USA). A tray of water was placed in the incubator to increase humidity. Spring water was continuously available in glass flasks with a paper towel wick. A 6- $\mathrm{cm}$ Petri dish containing a folded paper towel was wetted every 1-3 days to maintain high humidity. Bugs were fed either seeds of K. paniculata (F.W. Schumacher Co., Inc., Sandwich, Massachusetts, USA) or Cardiospermum halicacabum (Outsidepride.com, Inc., Independence, Oregon, USA). Due to the prevalence of cannibalism among hatchlings, eggs were removed and kept in Petri dishes until hatching, after which juveniles were removed to a separate container. Under these conditions, the generation time is 6-10 weeks. Ontogenetic allometry was assessed by raising bugs in isolation in 6-cm Petri dishes containing a folded paper towel, wetted daily, and exactly three seeds.

For the determination of reaction norms, soapberry bugs were collected from hatching dishes as second instars. Precise numbers of bugs and seeds of their natal host plant were combined in a terrarium or other container of known volume, with the water resources described above. Bugs were incubated at $26^{\circ} \mathrm{C}$ until all juveniles molted to adulthood or died. Water was replaced daily, but no food was removed or added. Dead bugs were removed periodically. All adults were scored for sex and morph, and in most treatments individuals were imaged as vouchers and for morphometric analysis.

\section{Morphometric analysis. The sizes and shapes of $J$. haematoloma wings were} analyzed using landmark-based geometric morphometric methods ${ }^{43}$ as implemented in the R package geomorph ${ }^{44}$. Bugs were anesthetized using $\mathrm{CO}_{2}$ and imaged on a trinocular stereo microscope (VWR International, Radnor, Pennsylvania, USA) with a Moticam 5 digital camera (Richmond, British Columbia, Canada). A millimeter-scale ruler was included in images to provide scale. Dorsal and ventral images of each specimen were recorded. Using ImageJ v1.46r5 linear pixel measurements were obtained for the lengths of body, labrum, labium, antennae, and femora of each leg. These values were then converted to metric distance using measurements of the scale. Twenty-four anatomical landmarks were placed on dorsal images of the wing (Fig. 1a, described in Supplementary Note 1). Generalized Procrustes analysis with partial Procrustes superimposition was preformed using minimized bending energy (Fig. 1b). Scaling coefficients of allometry were calculated from regression of log wing length to log head width relationships (Supplementary Figs. 1 and 3), as described by Huxley ${ }^{1}$. Modularity hypotheses were tested using a covariance ratio coefficient ${ }^{45}$ (Fig. 1c) in 1000 permutations. Morphological disparity within each morph was assessed using a permutation test of pairwise Procrustes distances with one million iterations. Tangent space positions for Procrustes-aligned specimens were determined along principal component axes (Fig. 1i). Procrustes ANOVA with permutation ${ }^{44}$ was used to assess hypotheses for patterns of shape variation among the aligned specimens, using 10,000 iterations.

Fecundity assessments. Fecundity was assessed for isolated, wild-caught $J$. haematoloma females and for individuals paired in crosses. In both cases, bugs were kept in plastic Petri dishes of $10 \mathrm{~cm}$ diameter and $2.5 \mathrm{~cm}$ deep. So that food availability did not limit female egg production, water and at least ten seeds of the natal host species were provided in uncovered $3.5 \mathrm{~cm}$ diameter Petri dishes. Seeds were replaced if they grew mold. Eggs were counted and removed within 7 days, before hatching. We could not control the age of wild-caught females. Therefore, we kept females in isolation and determined their rate of egg-laying after capture, from the first day eggs were produced until the last day eggs were produced.

Examination of testes and accessory glands. In an attempt to identify the mechanism for reduced fecundity in short-winged Cardiospermum-ecotype males, we examined the relative sizes of the testes and accessory glands from males of each ecotype. These organs were dissected from adult males at least 5 days old. The sizes of these organs were measured as their photomicrographic area.

Isolation of candidate genes. A reference transcriptome was prepared from 12 nascent adults of the Cardiospermum ecotype from Tavernier, FL, and 12 adults from $K$. paniculata in Aurora, CO. RNA was isolated from whole bodies and sent to Beckman Coulter Genomics (Danvers, Massachusetts) for poly-A selection, preparation of TruSeq libraries, and sequenced using Illumina HiSeq for $125 \mathrm{bp}$ paired-end reads. Reads from all samples were trimmed ${ }^{65}$ and assembled using Trinity ${ }^{66}$. The resulting transcriptome contained 258,322 contigs $\left(N_{50}=1596 \mathrm{bp}\right.$; $89.8 \%$ BUSCO score ${ }^{67}$ ). Reciprocal BLAST searching was used to identify sequences orthologous to candidate genes of interest. Fragments of the candidate genes, InR1, InR2, chico, Akt, and FoxO were isolated by PCR or constructed by commercial Gibson assembly (Integrated DNA Technologies, Inc., Coralville, Iowa). PCR primers are listed in Supplementary Table 7. PCR products were ligated into the pCR4-Topo vector (ThermoFisher Scientific, Waltham, Massachusetts) and transformed into One Shot TOP10 chemically competent cells (ThermoFisher Scientific). Plasmids were then isolated using the Purelink Quick Plasmid Miniprep kit (ThermoFisher Scientific) and sequences were confirmed by Sanger sequencing (Beckman Coulter Genomics). Sequences for Akt, Chico, and InR2 were obtained from assembled transcripts identified by BLAST homology and used for de novo Gibson assembly of linear DNAs (Integrated DNA Technologies, Inc.)

Orthology assignments. Most candidate genes had single orthologs in J. haematoloma that could be unambiguously identified using BLAST searches. However because of the possibility of confusion among members of the insulin receptor family, we determined orthology for these genes using phylogenetic inference. Predicted insulin receptor-like protein sequences were obtained from transcriptomes of three additional hemipterans, Jadera sanguinolenta, the box elder bug B. trivittata, and the milkweed bug O. fasciatus ${ }^{68}$, and from GenBank accessions for the fruit fly Drosophila melanogaster (P09208), the red flour beetle Tribolium castaneum (AHF20214; EFA02828), the pea aphid Acyrthosiphon pisum (XP_001952079; XP_001942660), and the brown planthopper N. lugens (AIY24638; AIY24639). Two putative insulin receptors were obtained from most of these species, except for D. melanogaster and J. sanguinolenta, from which only one InR was identified by BLAST. Four putative receptor tyrosine kinase sequences from B. trivittata and J. sanguinolenta were used as out-groups. Predicted amino acid sequences were aligned using ClustalW in Geneious 7.1.7 (Biomatters Ltd, Auckland, New Zealand) and manually trimmed to 1268 positions. The consensus protein tree was inferred using MrBayes v3.2.6. Amino acid substitution rates were modeled using the BLOSUM matrix, $\Gamma$-distributed rate variation across sites and a proportion of sites being invariable. Markov chain Monte Carlo analysis was run for 5 million generations with a $10 \%$ burn-in on a multi-processor core using 30 CPUs.

The consensus topology (Supplementary Fig. 7) strongly supports two paralogous groups, designated InR1 and InR2, following Xu et al. ${ }^{31}$. All insulin receptors were strongly monophyletic. The single InR protein from $D$. melanogaster did not nest with either paralog group. As only one functional insulin receptor has been identified in the fruit fly, it is possible that its position in this tree results from a unique history of selection. The single InR sequence from $J$. sanguinolenta was strongly supported as orthologous to InR1 from other species.

Gene expression. Gene expression and RNAi validations were determined using quantitative real-time RT-PCR (qPCR). For each gene, exact primers were designed using the Primer3 algorithm, avoiding conserved functional domains and dsRNA regions. Whole nascent adults were frozen in liquid nitrogen and stored at $-80^{\circ} \mathrm{C}$ before homogenization and total RNA extraction. If necessary, dissection was performed after flash freezing in RNAlater. Multiple biological replicates were included for each sex and morph. Using a poly-dT primer, complementary DNA was prepared from $1 \mu \mathrm{g}$ of total RNA using the iScript Select cDNA Synthesis Kit (BioRad, Hercules, California, USA). Gene expression was measured separately in the gonads and in the dorsal thorax, including forewings, hindwings, and flight muscle.

qPCR was performed using two methods: two-step SYBR green assays, including analysis of dissociation curves, and multiplex assays using dual-labeled probes. For the SYBR Green method, reactions used iTaq Universal SYBR Green Supermix (BioRad). Custom dual-labeled probes (Sigma-Aldrich, St. Louis, 
Missouri, USA) were used in reactions with iQ Multiplex Powermix (BioRad). All reactions were conducted on a CFX96 Touch qPCR System (BioRad). All assays included three to six DNA standards diluted from known concentrations of cloned or synthesized gene fragments with the addition of nonspecific salmon sperm DNA (ThermoFisher Scientific). For all tissues, we measured the expression of InR1, InR2, and FoxO, as well as the reference gene $\beta$-actin. For dorsal thorax samples, we also measured expression of Distal-less (Dll); vitellogenin (vit) expression was measured in gonad samples. Assays were normalized using the expression of $\beta$ actin. For validation of RNAi, expression was compared between gene-specific and nonspecific GFP control dsRNA treatments. All analyses were performed using the means of technical triplicates and included a minimum of three biological replicates for each group under comparison.

RNA interference. Gene function was tested during juvenile-to-adult development in J. haematoloma using RNAi. A DNA template was amplified from gene fragments using primers with a 20 -nucleotide T7 viral promoter sequence at the 5 '-end. This linear DNA was used as a template in bidirectional RNA synthesis using the MegaScript T7 transcription kit (ThermoFisher Scientific). The product was treated with DNase I to remove template DNA, then annealed by cooling and purified by precipitation in cold ammonium acetate and ethanol. After resuspension in nuclease-free water, dsRNA concentrations were determined using a nanoscale spectrophotometer (GE Life Sciences NanoVue) and diluted to $2 \mu \mathrm{g} / \mu \mathrm{l}$. Buffered dsRNA was injected into fourth instar J. haematoloma. Bugs were anesthetized using $\mathrm{CO}_{2}$. Approximately $0.2 \mu \mathrm{l}$ of $2 \mu \mathrm{g} / \mu \mathrm{l}$ dsRNA was injected into the ventral abdomen using a pulled-glass capillary needle. Nonspecific GFP sequence was used as a control for the potential effects of injection wounding and nonspecific dsRNA toxicity. Knockdown of gene activity was confirmed using qRT-PCR (Supplementary Fig. 9).

Insulin treatment. Exogenous insulin treatments were based on previous experiments from two Lepidoptera, Manduca sexta ${ }^{53}$ and Bombyx mori ${ }^{54}$. Bovine pancreatic insulin (Sigma-Aldrich) was prepared in saline buffer at $160 \mathrm{ng} / \mu \mathrm{l}$. Injection was made into the abdomen of fourth instars, in the same manner used for introduction of dsRNA. This method delivered roughly $0.5 \mathrm{ng}$ insulin per $\mathrm{mg}$ body weight.

Statistical analysis. All statistical tests were conducted in R (version 3.4.3). Plots were also generated using $\mathrm{R}$, with some modifications to graphic layout being made using Illustrator CC 2015 (Adobe Systems, San Jose, California). Details of statistical analyses are given in the Supplementary Note 1. Supplementary Data File 1 includes $\mathrm{R}$ markdown code to reproduce each statistical test and the original plots.

Code availability. All analysis scripts, including $\mathrm{R}$ code used for the production of the main and supplementary plots, are available in Supplementary Data File 1.

Data availability. Gene sequences are archived under GenBank accession numbers MF620038 to MF620048. All other data that support the findings of this study are archived in Supplementary Data File 1.

Received: 7 January 2017 Accepted: 29 March 2018

Published online: 27 April 2018

\section{References}

1. Huxley, J. S. Problems of Relative Growth. (Dial Press, New York, 1932).

2. Levins, R. Evolution in changing environments: some theoretical explorations (Princeton Univ. Press, Princeton, New Jersey, USA, 1968).

3. West-Eberhard, M. J. Developmental Plasticity and Evolution (Oxford Univ. Press, Oxford, 2003).

4. Stearns, S. C. The evolutionary significance of phenotypic plasticity. Bioscience 39, 436-445 (1989).

5. Roff, D. A. The evolution of threshold traits in animals. Q. Rev. Biol. 71, 3-35 (1996).

6. Moran, N. A. The evolutionary maintenance of alternative phenotypes. Am. Nat. 139, 971-989 (1992).

7. Woltereck, R. Weitere experimentelle Untersuchungen über Artveränderung, speziel über das Wesen quantitativer Artunterschiede bei Daphniden. Verh. der Dtsch. Zool. Ges. 19, 110-173 (1909).

8. Whitman, D. W. \& Agrawal, A. A. in Phenotypic Plasticity of Insects: Mechanisms and Consequences (eds Whitman, D. W. \& Ananthakrishnan, T. N.) 1-63 (Science Publishers, Enfield, New Hampshire, USA, 2009)

9. Moczek, A. P. \& Nijhout, H. F. Developmental mechanisms of threshold evolution in a polyphenic beetle. Evol. Dev. 4, 252-264 (2002).
10. Brisson, J. A. \& Stern, D. L. Common genome-wide patterns of transcript accumulation underlying the wing polyphenism and polymorphism in the pea aphid (Acyrthosiphon pisum). Evol. Dev. 9, 338-346 (2007).

11. Zera, A. J. in Phenotypic Plasticity of Insects: Mechanisms and Consequences (eds Whitman, D. W. \& Ananthakrishnan, T. N.) 609-653 (Science Publishers, Enfield, New Hampshire, USA, 2009).

12. Denver, R. J. Neuroendocrinology of amphibian metamorphosis. Curr. Top. Dev. Biol. 103, 195-227 (2013).

13. Gotoh, H. et al. Developmental link between sex and nutrition; doublesex regulates sex-specific mandible growth via juvenile hormone signaling in stag beetles. PLoS Genet. 10, e1004098 (2014).

14. Borthwick, H. A. \& Downs, R. J. Roles of active phytochrome in control of flowering of Xanthium pensylvanicum. Bot. Gaz. 125, 227-231 (1964).

15. Kijimoto, T. \& Moczek, A. P. Hedgehog signaling enables nutrition-responsive inhibition of an alternative morph in a polyphenic beetle. Proc. Natl Acad. Sci. USA 113, 5982-5987 (2016)

16. Brakefield, P. M., Kesbeke, F. \& Koch, P. B. The regulation of phenotypic plasticity of eyespots in the butterfly Bicyclus anynana. Am. Nat. 152, 853-860 (1998).

17. Clausen, J., Keck, D. D. \& Hiesey, W. M. Experimental Studies on the Nature of Plant Species. I. Effect of Varied Environments on Western North American Plants. 520, pp.vii +452 pp (Carnegie Instituton of Washington Publication, Washington DC, 1940).

18. Bradshaw, A. D. Evolutionary significance of phenotypic plasticity in plants. Adv. Genet. 13, 115-155 (1965).

19. Schlichting, C. D. The evolution of phenotypic plasticity in plants. Annu. Rev. Ecol. Syst. 17, 667-693 (1986).

20. Gibson, G. \& Hogness, D. S. Effect of polymorphisms in the Drosophila regulatory gene Ultrabithorax on homeotic stability. Science 271, 200-203 (1996).

21. Roskam, J. C. \& Brakefield, P. M. A comparison of temperature-induced polyphenism in African Bicylus butterflies from a seasonal Savannahrainforest ecotone. Evolution 50, 2360-2372 (1996).

22. Losos, J. B. et al. Evolutionary implications of phenotypic plasticity in the hindlimb of the lizard Anolis sagrei. Evolution 54, 301-305 (2000).

23. Lind, M. I. \& Johansson, F. The degree of adaptive phenotypic plasticity is correlated with the spatial environmental heterogeneity experienced by island populations of Rana temporaria. J. Evol. Biol. 20, 1288-1297 (2007).

24. Greene, E., Canfield, M. \& Ehmer, A. in Phenotypic Plasticity of Insects: Mechanisms and Consequences (eds Whitman, D. W. \& Ananthakrishnan, T. N.) 135-146 (Science Publishers, Enfield, New Hampshire, USA, 2009).

25. Ghalambor, C. K. et al. Non-adaptive plasticity potentiates rapid adaptive evolution of gene expression in nature. Nature 525, 372-375 (2015).

26. Brakefield, P. M. \& Larsen, T. B. The evolutionary significance of dry and wet season forms in some tropical butterflies. Biol. J. Linn. Soc. 22, 1-12 (1984).

27. Harrison, R. G. Dispersal polymorphisms in insects. Annu. Rev. Ecol. Syst. 11, 95-118 (1980).

28. Shingleton, A. W. \& Frankino, W. A. The (ongoing) problem of relative growth. Curr. Opin. Insect Sci. 25, 9-19 (2018).

29. Zera, A. J. \& Denno, R. F. Physiology and ecology of dispersal polymorphism in insects. Annu. Rev. Entomol. 42, 207-230 (1997).

30. Brisson, J. A. \& Davis, G. K. The right tools for the job: regulating polyphenic morph development in insects. Curr. Opin. Insect Sci. 13, 1-6 (2015).

31. Xu, H.-J. et al. Two insulin receptors determine alternative wing morphs in planthoppers. Nature 519, 464-467 (2015).

32. Emlen, D. J., Szafran, Q., Corley, L. S. \& Dworkin, I. Insulin signaling and limb-patterning: candidate pathways for the origin and evolutionary diversification of beetle 'horns'. Heredity 97, 179-191 (2006).

33. Snell-Rood, E. C. \& Moczek, A. P. Insulin signaling as a mechanism underlying developmental plasticity: the role of FOXO in a nutritional polyphenism. PLoS One 7, e34857 (2012).

34. Nijhout, H. F. \& McKenna, K. Z. The distinct roles of insulin signaling in polyphenic development. Curr. Opin. Insect Sci. 25, 58-64 (2018).

35. Tsai, J. -F., Hsieh, Y. -X. \& Rédei, D. The soapberry bug, Jadera haematoloma (Insecta, Hemiptera, Rhopalidae): first Asian record, with a review of bionomics. Zookeys 297, 1-41 (2013).

36. Carroll, S. P. \& Boyd, C. Host race radiation in the soapberry bug: natural history with the history. Evolution 46, 1053-1069 (1992).

37. Carroll, S. P., Dingle, H. \& Klassen, S. P. Genetic differentiation of fitnessassociated traits among rapidly evolving populations of the soapberry bug. Evolution 51, 1182-1188 (1997)

38. Carroll, S. P., Klassen, S. P. \& Dingle, H. Rapidly evolving adaptations to host ecology and nutrition in the soapberry bug. Evol. Ecol. 12, 955-968 (1998).

39. Winchell, R., Dingle, H. \& Moyes, C. D. Enzyme profiles in two wing polymorphic soapberry bug populations (Jadera haematoloma: Rhopalidae). J. Insect Physiol. 46, 1365-1373 (2000).

40. Cenzer, M. L. Adaptation to an invasive host is driving the loss of a native ecotype. Evolution 70, 2296-2307 (2016). 
41. Dingle, H. \& Winchell, R. Juvenile hormone as a mediator of plasticity in insect life histories. Arch. Insect Biochem. Physiol. 35, 359-373 (1997).

42. Cenzer, M. L. Maladaptive plasticity masks the effects of natural selection in the red-shouldered soapberry bug. Am. Nat. 190, 521-533 (2017).

43. Bookstein, F. L. Morphometric Tools for Landmark Data: Geometry and Biology (Cambridge Univ. Press, Cambridge, UK, 1991).

44. Adams, D. C., Otárola-Castillo, E. \& Paradis, E. geomorph: an R package for the collection and analysis of geometric morphometric shape data. Methods Ecol. Evol. 4, 393-399 (2013).

45. Adams, D. C. Evaluating modularity in morphometric data: challenges with the RV coefficient and a new test measure. Methods Ecol. Evol. 7, 565-572 (2016).

46. Murren, C. J. et al. Constraints on the evolution of phenotypic plasticity: limits and costs of phenotype and plasticity. Heredity 115, 293-301 (2015).

47. Roff, D. A. The evolution of flightlessness in insects. Ecol. Monogr. 60, 389-421 (1990).

48. Kramer, J., Davidge, J., Lockyer, J. \& Staveley, B. Expression of Drosophila FOXO regulates growth and can phenocopy starvation. BMC Dev. Biol. 3, 5 (2003).

49. Tang, H. Y., Smith-Caldas, M. S. B., Driscoll, M. V., Salhadar, S. \& Shingleton, A. W. FOXO regulates organ-specific phenotypic plasticity in Drosophila. PLoS Genet. 7, e1002373 (2011).

50. Miron, M. et al. The translational inhibitor $4 \mathrm{E}-\mathrm{BP}$ is an effector of $\mathrm{PI}(3) \mathrm{K} / \mathrm{Akt}$ signalling and cell growth in Drosophila. Nat. Cell Biol. 3, 596-601 (2001).

51. Jünger, M. A. et al. The Drosophila Forkhead transcription factor FOXO mediates the reduction in cell number associated with reduced insulin signaling. J. Biol. 2, 20 (2003).

52. Puig, O., Marr, M. T., Ruhf, M. L. \& Tjian, R. Control of cell number by Drosophila FOXO: downstream and feedback regulation of the insulin receptor pathway. Genes Dev. 17, 2006-2020 (2003).

53. Koyama, T., Syropyatova, M. O. \& Riddiford, L. M. Insulin/IGF signaling regulates the change in commitment in imaginal discs and primordia by overriding the effect of juvenile hormone. Dev. Biol. 324, 258-265 (2008)

54. Gu, S.-H., Lin, J.-L., Lin, P.-L. \& Chen, C.-H. Insulin stimulates ecdysteroidogenesis by prothoracic glands in the silkworm Bombyx mori. Insect Biochem. Mol. Biol. 39, 171-179 (2009).

55. Kromer-Metzger, E. \& Lagueux, M. Expression of the gene encoding an insulin-related peptide in Locusta (Insecta, Orthoptera). Eur. J. Biochem. 221, 427-434 (1994).

56. Masumura, M., Satake, S., Saegusa, H. \& Mizoguchi, A. Glucose stimulates the release of bombyxin, an insulin-related peptide of the silkworm Bombyx mori. Gen. Comp. Endocrinol. 118, 393-399 (2000)

57. Johnston, L. A. \& Gallant, P. Control of growth and organ size in Drosophila. Bioessays 24, 54-64 (2002).

58. Shingleton, A. W., Das, J., Vinicius, L. \& Stern, D. L. The temporal requirements for insulin signaling during development in Drosophila. PLoS Biol. 3, 1607-1617 (2005).

59. Nijhout, H. F. The control of body size in insects. Dev. Biol. 261, 1-9 (2003).

60. Gokhale, R. H., Hayashi, T., Mirque, C. D. \& Shingleton, A. W. Intra-organ growth coordination in Drosophila is mediated by systemic ecdysone signaling. Dev. Biol. 418, 135-145 (2016).

61. Lavine, L. C. et al. Cloning and characterization of an mRNA encoding an insulin receptor from the horned scarab beetle Onthophagus nigriventris (Coleoptera: Scarabaeidae). Arch. Insect Biochem Physiol. 82, 43-57 (2013).

62. Lin, X. \& Lavine, L. C. Endocrine regulation of a dispersal polymorphism in winged insects: a short review. Curr. Opin. Insect Sci. 25, 20-24 (2018).

63. Misof, B., Liu, S., Meusemann, K., Peters, R. S. \& Al., E. Phylogenomics resolves the timing and pattern of insect evolution. Science 346, 763-767 (2014).

64. Emlen, D. J., Warren, I. A., Johns, A., Dworkin, I. \& Lavine, L. C. A mechanism of extreme growth and reliable signaling in sexually selected ornaments and weapons. Science 337, 860-864 (2012).
65. Bolger, A. M., Lohse, M. \& Usadel, B. Trimmomatic: a flexible trimmer for Illumina sequence data. Bioinformatics 30, 2114-2120 (2014).

66. Grabherr, M. G. et al. Full-length transcriptome assembly from RNA-Seq data without a reference genome. Nat. Biotechnol. 29, 644-652 (2011).

67. Simão, F. A., Waterhouse, R. M., Ioannidis, P., Kriventseva, E. V. \& Zdobnov, E. M. BUSCO: assessing genome assembly and annotation completeness with single-copy orthologs. Bioinformatics 31, 3210 (2015).

68. Panfilio, K. A. et al. Molecular evolutionary trends and feeding ecology diversification in the Hemiptera, anchored by the milkweed bug genome. bioRxiv 201731 (2017). https://doi.org/10.1101/201731

\section{Acknowledgements}

We thank Randall Downer, Margaret McKeon, Joshua L. Steele, and Serena M. Graham for technical assistance. Field assistance was generously provided by Robert Angelini, Linda Angelini, and Serena M. Graham. Thanks to Kristen Panfilio, Iris Vargas Jentzsch, Joshua Benoit, and Stephen Richards for sharing transcriptome data from O. fasciatus before publication. Scott Carroll provided comments on an early draft of the manuscript Research reported in this publication was supported by the Colby College Division of Natural Sciences, by an Institutional Development Award (IDeA) from the National Institute of General Medical Sciences of the National Institutes of Health under grant number P20GM0103423, and by grant IOS-1350207 from National Science Foundation to DRA.

\section{Author contributions}

D.R.A. designed and supervised all experiments, conducted the analyses, and wrote the manuscript. Data on J. haematoloma distributions was gathered by D.R.A., M.C., M.C.P., and M.M.F. Editing of the manuscript was done by M.M.F., M.C.P., E.M.R., W.S.H., L.C., M.C., and D.R.A. M.M.F., M.C.P., A.E.T., E.M.R., J.C.V., J.S.S., W.S.H., W.R.S., L.C., and D.R.A. conducted portions of the experiments.

\section{Additional information}

Supplementary Information accompanies this paper at https://doi.org/10.1038/s41467 018-04102-1.

Competing interests: The authors declare no competing interests.

Reprints and permission information is available online at http://npg.nature.com/ reprintsandpermissions/

Publisher's note: Springer Nature remains neutral with regard to jurisdictional claims in published maps and institutional affiliations.

Open Access This article is licensed under a Creative Commons Attribution 4.0 International License, which permits use, sharing, adaptation, distribution and reproduction in any medium or format, as long as you give appropriate credit to the original author(s) and the source, provide a link to the Creative Commons license, and indicate if changes were made. The images or other third party material in this article are included in the article's Creative Commons license, unless indicated otherwise in a credit line to the material. If material is not included in the article's Creative Commons license and your intended use is not permitted by statutory regulation or exceeds the permitted use, you will need to obtain permission directly from the copyright holder. To view a copy of this license, visit http://creativecommons.org/ licenses/by/4.0/

(C) The Author(s) 2018 